\title{
DISTRIBUIÇÃO DE Panstrongylus megistus (HEMIPTERA, REDUVIIDAE, TRIATOMINAE) NO ESTADO DO MARANHÃO, BRASIL
}

\author{
José Manuel Macário REBÊLO
}

\begin{abstract}
RESUMO - Apresenta-se a distribuição de Panstrongylus megistus no Estado do Maranhão, com base nos dados do inquérito triatominico realizado pela Fundação Nacional de Saúde, de 1982 a 1995. O método básico utilizado foi a captura de adultos e ninfas nos ambientes peri e intradomiciliares. Os trabalhos de coleta dentro das casas começavam pelos cômodos dos fundos, passando de um aposento para o outro, até a frente. Na parte externa das casas foram inspecionados pátio, quintal, jardim e anexos - galinheiro, chiqueiro, estábulo, curral etc. Todos os exemplares encontrados eram capturados, identificados e, quando possível, examinado o conteúdo intestinal para detecção de Trypanosoma. O estudo envolveu 87 dos 136 municípios em que se divide geograficamente o Maranhão. O Panstrongylus megistus encontrava-se confinado em 14 municipios pertencentes à zona dos cerrados que caracterizam a porção mais meridional do Estado. Dos 1.632 exemplares capturados 277 foram examinados, resultando no indice global de infecção por Trypanosoma tipo cruzi de 1,1\%. Os municipios que apresentaram maior quantidade de espécimens capturados foram São João dos Patos (22,4\%), Pastos Bons (15\%), São Raimundo das Mangabeiras (14,5\%), Mirador (14,3\%), Riachão (8,3\%) e Loreto (6,2\%). Atualmente, o P. megistus restringe a sua área de ocorrência às regiões sudeste e sul do Maranhão. A julgar por este padrão de distribuição, presume-se que esta espécie penetrou no território maranhense através dos estados do Piaui, Goiás ou Tocantins, utilizando os cerrados como rota.
\end{abstract}

Palavras-chave: Triatomíneos, Panstrongylus megistus, distribuição, doença de Chagas, Maranhão.

Distribution of Panstrongylus megistus (Hemiptera, Reduviidae, Triatominae) in Maranhão State, Brazil.

ABSTRACT - Based on a survey on the presence of $P$. megistus in several localities of Maranhão State, performed by the National Foundation of Health, from 1982 to 1995, the distribution of this species is shown. The capture of the adults and nymphs in the domestic environment was the basic method employed. The process of capture started at the rear and finished at the front of the human dwellings, and the surroundings as patio, back yard, garden, harbouring chickens, were inspected afterwards. All the specimens collected were identified. An effort was made to try to detect Trypanosoma from the intestinal content of some bugs. Maranhão is made up of 136 municipalities and the survey covered 87 of them. Panstrongylus megistus was in 14 municipal districts which belonging to the savana zone (cerrados) in the southern portion of the State. In total, 1,632 specimens of $P$. megistus were captured and 277 were examined resulting in the rate of $1.1 \%$ of infection with Trypanosoma cruzi. São João dos Patos (22.4\%), Pastos Bons (15\%), São Raimundo das Mangabeiras (14.5\%), Mirador (14.3\%), Riachão (8.3\%) and Loreto (6.2\%) were the municipal districts in which the presence of $P$. megistus was more common. For the moment, $P$. megistus is found only in southeast and south of Maranhão. Judging by the current distribution this specie penetrated through the "cerrados" from of the neighbouring states of Goiás, Tocantins and Piaui.

Key-words: Triatominae, Panstrongylus megistus, Distribution, Chagas disease, Maranhão

\section{INTRODUÇÃO}

O padrão de distribuição dos triatomíneos vetores da doença de Chagas no Brasil é relativamente bem conhecido, sendo poucos os Estados que ainda necessitam de um estudo mais aprofundado. A evolução do conhecimento sobre sua biologia, origem e distribuição geográfica foi

1 Núcleo de Patologia Tropical e Medicina Social. Departamento de Patologia/UFMA. Praça Madre Deus № 2, CEP 65.025-560, São Luís, MA. 
sintetizada nos trabalhos de Sherlock (1979), Lent \& Wygodzisky (1979) e Forattini (1980), e as características ecofisiológicas foram descritas por Zeledon (1983).

O Panstrongylus megistus é considerado um dos mais importantes vetores da doença de Chagas no Brasil, devido à sua ampla distribuição geográfica. Tem sido encontrado nos seguintes Estados: Maranhão, Piaui, Ceará, Rio Grande do Norte, Paraíba, Pernambuco, Alagoas, Sergipe, Bahia, Espírito Santo, Rio de Janeiro, São Paulo, Paraná, Santa Catarina, Rio Grande do Sul, Minas Gerais, Goiás e Mato Grosso do Sul, especialmente associado a ambientes úmidos e quentes (Alencar, 1987; Espínola \& Diotaiuti, 1995). De São Paulo para o sul diminui sua densidade intradomiciliar e sua importância vetorial. Costuma ser encontrado ao longo da Mata Atlântica e em áreas restritas do Uruguai, Argentina, Paraguai e Bolivia (Sherlock, 1979).

Depois do Triatoma infestans, é a espécie com maior capacidade de domiciliação (Dias, 1987), apresentando diferentes comportamentos ecológicos nas regiões Nordeste e Sudeste do Brasil (Nascimento et al., 1997). Encontra-se domiciliada no Ceará e na Bahia, sendo silvestre em Santa Catarina, com comportamento intermediário entre estas duas regiões no sul de Minas Gerais e norte de São Paulo, no vale do rio Grande. Atualmente, a espécie acha-se muito restrita a focos peridomiciliares e a invasões eventuais de casas, em áreas de vigilância, onde se aloja em montes de tijolos, paredes emburacadas e galinheiros (Dias \& Coura,1997).
No Maranhão, a distribuição do $P$. megistus é atualmente conhecida em virtude dos sucessivos inquéritos triatomínicos realizados em diversos municipios durante o inquérito nacional (Silveira et al., 1984) e posteriormente nos trabalho de rotina da FNS (Rebêlo et al., 1998).

Segundo Alencar (1987) só se pode caracterizar o território geográfico de uma espécie de triatomíneo quando se encontram colônias, isto é, adultos e ninfas em número significativo. Neste contexto, não se dispõe de dados consistentes relativos ao Estado, de modo que o objetivo deste trabalho é simplesmente mapear os municípios nos quais esta espécie de triatomíneo tem sido encontrada, indicando aqueles em que sua ocorrência tem sido mais freqüente.

\section{MATERIAL E MÉTODOS}

De 1982 a 1995 a Fundação Nacional de Saúde realizou vários inquéritos entomológicos para detectar a presença de triatomíneos no Estado do Maranhão. O método básico para a execução dos inquéritos foi a captura de adultos e ninfas nos ambientes peri e intradomiciliar. A inspeção iniciava dentro das casas, começando pelos cômodos dos fundos e passando de um aposento para o outro, até à frente, circulando-se em cada um deles pela direita. Terminada a visita interna, prosseguia-se a inspeção na parte externa (pátio, quintal, jardim e anexos galinheiro, chiqueiro, estábulo, curral etc), seguindo sempre pela direita. Este método foi adaptado daquele utilizado no Programa de Controle da Febre Amarela (MS, 1989). Todos os 
exemplares vivos encontrados eram capturados com pinça, depositados em recipientes adequados para o transporte até o laboratório, onde eram identificados especificamente e, quando possível, examinado o conteúdo intestinal para detecção de Trypanosoma.

Os dados organizados no setor de estatística da FNS-MA relaciona a quantidade de espécimens encontrada em cada município inspecionado sem fazer distinção entre machos, fêmeas e formas imaturas.

O estudo foi realizado em 87 dos 136 municípios que compõem o Estado do Maranhão. Para efeito de discussão agruparam-se os municipios em 7 zonas fitogeográficas, ficando os 136 municipios assim distribuidos: Cerrados meridionais (25 municípios), Amazônia (18), Cerrado e Caatinga (8), Dunas, Mangue e Restinga (16), Campo Aluvial
Flúvio Marinho (28), Matas, Cocais e Cerrados (18), Matas e Cocais (23).

\section{RESULTADOS E DISCUSSÃO}

No total, foram capturados 1.632 exemplares de $P$. megistus, encontrados em 14 municípios (Tab. 1), todos situados na porção meridional do Estado (Fig. 1). Os municípios que apresentaram maior quantidade de espécimens capturados no período de estudo foram São João dos Patos $(22,4 \%)$, Pastos Bons (15\%), São Raimundo das Mangabeiras (14,5\%), Mirador (14,3\%), Riachão $(8,3 \%)$ e Loreto $(6,2 \%)$. Os demais municipios representaram juntos $19,3 \%$ da amostra total.

A região meridional do Estado, na qual $P$. megistus vem sendo encontrado, apresenta em sua fisionomia ecológica o cerrado como vegetação dominante, caracterizado por possuir árvores tortuosas, com grandes folhas e por

Tabela 1. Números de espécimens de Panstrongylus megistus capturados no Estado do Maranhão, de 1982 a 1995, e indice de infecção por Trypanosoma.

\begin{tabular}{lcccc}
\hline Municípios & Capturados & Examinados & Positivos & $(\%)$ \\
\hline Barão do Grajaú & 37 & & & \\
Buriti Bravo & 20 & & & \\
Colinas & 61 & & & \\
Loreto & 102 & 36 & & 0,8 \\
Mirador & 234 & 128 & 1 & \\
Paraibano & 30 & & & \\
Passagem Franca & 42 & & 2 & 2,0 \\
Pastos Bons & 245 & 100 & & \\
Riachão & 135 & & & \\
São Félix de Balsas & 24 & 11 & & \\
São Francisco do Maranhão & 27 & & & \\
Săo João dos Patos & 365 & 2 & & \\
São Raimundo das Mangabeiras & 236 & & & \\
Sucupira do Norte & 74 & & & \\
\hline Total & 1632 & 277 & & \\
\hline
\end{tabular}




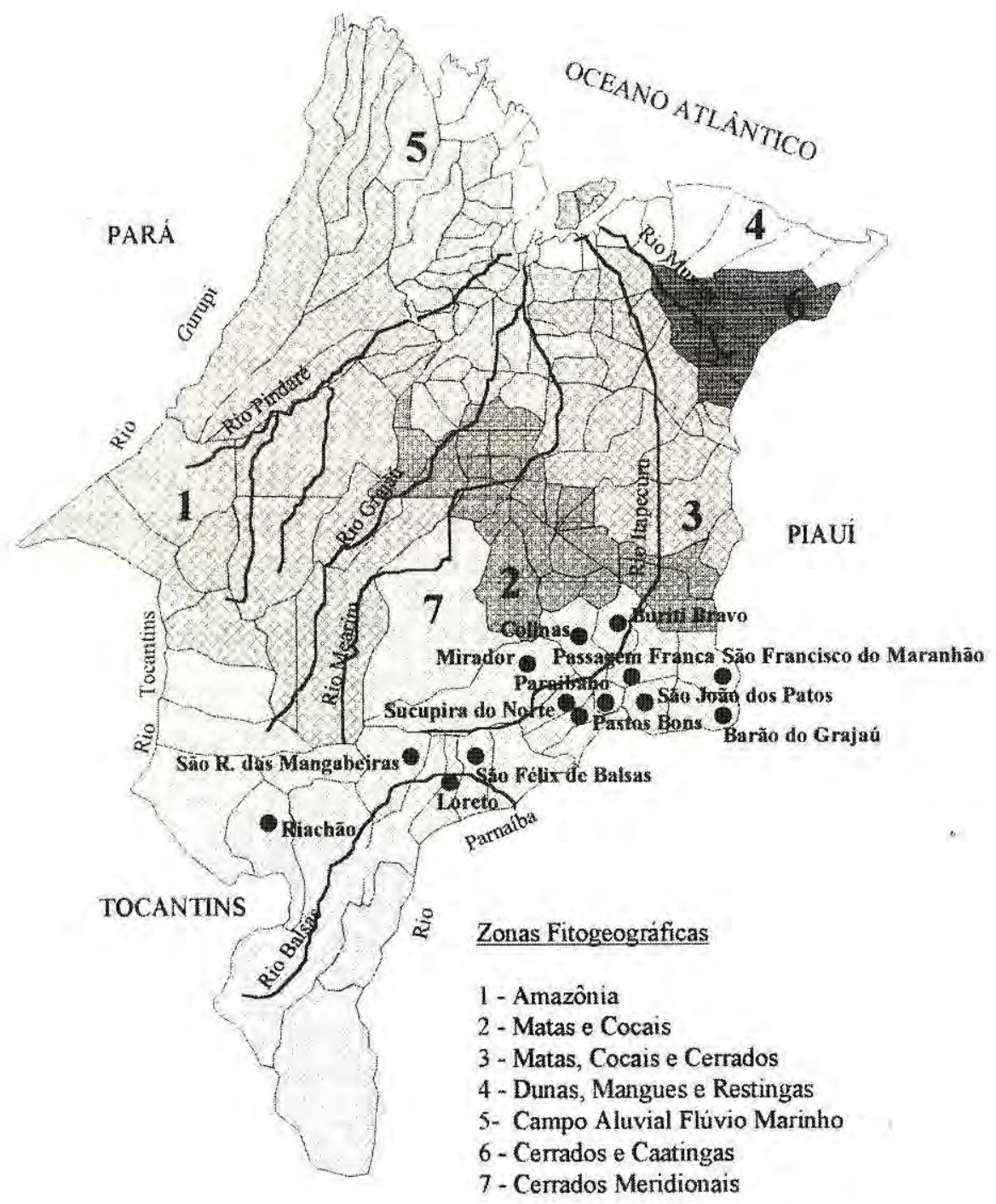

Figura 1. Mapa do Estado do Maranhão dividido em zonas fitogeográficas.

- Municipios positivos para Panstrongylus megistus de 1982 a 1995. 
formas biológicas típicas, adaptadas a solos deficientes e profundos. Predominam nessa formação os seguintes vegetais: sambaíba (Curatella americana), murici (Byrsonima crassifolia), faveira (Platypodium elegans), piqui (Caryocar brasiliensis), jatobá (Hymenaea courbaril) e andiroba (Carapa guianensis). É uma grande fitorregião que se estende por sobre 0 topo dos extensos chapadões desde o alto curso do rio Parnaíba, no extremo sul do Maranhão, até a cabeceira do rio Itapecuru, no centro do Estado, e para sudeste avança até a região do médio Parnaíba (IBGE, 1984).

Nas nascentes dos rios Grajaú, Mearim e Itapecuru, os cerrados são influenciados pelo clima quente semiúmido; e nos altos cursos dos rios Balsas e Parnaíba, o domínio é do clima quente semi-árido (IBGE, 1984).

É notório o confinamento do $P$. megistus nos municípios do sul do Estado, uma região de altitude, clima árido, cerrado e, talvez, a rota de entrada deste hemíptero no Maranhão, oriundo de Estados vizinhos (Goiás, Tocantins ou Piaui). Nesta zona podem-se encontrar outras espécies de triatomíneos, como P. lignarius, $T$. brasiliensis, T. pseudomaculata, Rhodnius nasustus, $R$. neglectus, $R$. pictipes e $R$. robustus (Rebêlo et al., 1998), mas o P. megistus é a espécie mais comum. Aliás, o domínio deste barbeiro no sul do Maranhão vem sendo observado desde os estudos realizados no final da década de 70 , por ocasião do inquérito nacional, quando chegou a representar $70 \%$ dos espécimens capturados nos anos de 1977 e 1978 (FNS, 1984).

Convém ressaltar que além do sul do Maranhão apresentar fronteiras com Estados onde a doença de Chagas é dita endêmica, possui também condições ambientais, sócio-econômicas e culturais para a adaptação de $P$. megistus, devido a existência de casas de má qualidade, não rebocadas, com teto de palha, possibilitando a colonização no intra domicílio e, conseqüentemente, o estabelecimento do ciclo da infecção nas casas.

De acordo com o relatório da FNS (1990), em 1978 foram trabalhados naquela área 21 municípios nas atividades de pesquisa e captura, com 5.310 localidades e 101.353 unidades domiciliares. Das localidades trabalhadas, 113 foram positivas, com apenas 171 prédios abrigando barbeiros, na maioria colonizados. Foram capturados 672 triatomíneos, apenas 15 deles positivos para T. cruzi, predominando a espécie $P$. megistus. Os municípios que apresentaram, na época, prevalência acima de $5 \%$ foram Colinas, São Félix de Balsas, Barão de Grajaú, Sucupira do Norte, Loreto, Mirador, Pastos Bons e São João dos Patos, todos situados no sudeste e sul do Estado. A situação vetorial pouco modificou nas duas últimas décadas, pois esses municípios, além de São Raimundo das Mangabeiras e Riachão, continuam apresentando indices de infestação elevados. A propósito, em 1981 tomou-se conhecimento dos resultados do inquérito sorológico que acusava a situação particular de São Raimundo 
das Mangabeiras, com uma prevalência de $6,2 \%$ e com todos os soropositivos considerados casos autóctones (FNS, 1989).

Então, do ponto de vista vetorial de $P$. megistus, o eixo sudeste-sul seria a região de grande importância na transmissão da doença de Chagas. À medida que diminui a latitude, os cerrados misturam-se com florestas úmidas e cocais (Orbignya phalerata). Há um total desaparecimento de $P$. megistus, que passa a ser substituído por outras espécies de Panstrongylus e do gênero Rhodnius. Assim, com os dados de que se dispõe atualmente, o limite norte da distribuição de $P$. megistus no Maranhão está nos municípios de Mirador e Passagem Franca; ao sul e oeste está o município de Riachão e a leste o município de São Francisco do Maranhão. Contudo, deve-se ressaltar que os cerrados característicos do sul do Estado avançam em direção ao centro e a nordeste da Unidade Federativa em áreas climáticas que se alternam entre semi-úmidas e semi-áridas, oferecendo condições fitogeográficas e climáticas não muito diferentes daquela onde este hemíptero vem sendo encontrado. Neste aspecto, é possivel que se venha encontrálo nas áreas mistas de cerrados, caatingas, cocais e matas, desde de que se faça uma busca mais intensiva, com esforço mais adequado. Enquanto isso, os municipios mais infestados são, justamente, aqueles situados a sudeste do Estado, limitrofes com o Estado do Piauí.

Infelizmente foram poucos os registros de espécimens de $P$. megistus submetidos ao exame de infecção por
Trypanosoma. Do total de 1.632 indivíduos capturados, somente 277 foram examinados, resultando em $1,1 \%$ de exemplares infectados por Trypanosoma tipo cruzi, um índice considerado baixo se comparado com aquele obtido no Ceará (7\%). De acordo com Alencar (1987), a taxa que se pode considerar como característica dos Estados do Nordeste, que compõem a área de clima mais seco e árido, é de $2 \%$. Dos 18 municípios em que se encontrou a espécie, apenas Mirador e Pastos Bons apresentaram exemplares naturalmente infectados. Em virtude da fragilidade dos dados obtidos junto à FNS-MA, há necessidade da realização de novos inquéritos triatominicos, mais consistentes, para se ter uma noção mais realista da situação da distribuição não só deste barbeiro, como dos triatomineos de um modo geral e de sua infecção natural por $T$. cruzi com vista ao melhor controle da doença de Chagas no Estado do Maranhão.

\section{AGRADECIMENTO}

O autor agradece à Fundação Nacional de Saúde pela concessão dos dados aqui analisados.

\section{Bibliografia citada}

Alencar, J.E. 1987. História natural da doença de Chagas no Estado do Ceará. Fortaleza, Imprensa Universitária da UFC. 341 p.

Dias J.C.P. 1987. Control of Chagas disease in Brazil. Parasitol. Today, 3: 336-341.

Dias J.C.P.; Coura J.R. 1997. Epidemiologia. In: Dias/Coura (eds.) Clinica e terapêutica da doença de Chagas: uma abordagem prática para clinico geral. Rio de Janeiro, Fiocruz. p. 33-65. 
Espínola, H.N.; Diotaiuti, L. 1995. Hemiptera. In: Neves D.P. (ed.) Parasitologia Humana. São Paulo, Atheneu. p.346-366.

Forattini O. P. 1980. Biogeografia, origem e distribuição da domiciliação de triatomineos no Brasil. Rev. Saúde Púb. 14: 265-299.

Fundação Nacional de Saúde-Ministério da Saúde. Programa de Controle da Doença de Chagas. Relatório Anual do Serviço de Epidemiologia. São Luis, 1984, 1989, 1990.

Instituto Brasileiro de Geografia e Estatistica. 1984. Atlas do Maranhão. Superintendência de Estudos Geográficos e Sócio-Econômicos. Rio de Janeiro. 104p.

Lent, H.; Wygodzinsky, P. 1979. Revision of the Triatominae (Hemiptera, Reduviidae), and their significance as vectors of Chagas disease, Bull. Am. Museum Nat. History, 163 (3): $127-520$.

Ministério da Saúde. Superintendência de Campanhas de Saúde Pública-Sucam. 1989. Combate ao Aedes aegypti. Instrução para guardas, guardas chefes e inspetores. Gráfica e Editora Itamarati Itda. Brasília. 92p.
Nascimento, C.; Marassá, AM., Curado, I.; Piazza, R.M.F. 1997. Encontro de Panstrongylus megistus $\mathrm{em}$ ecótopo artificial: domiciliação ou mera visitação?. Rev. Soc. Bras. Med. Trop., 30 (4): 333-336.

Rebêlo, J.M.M., Barros, V.L.L.; Mendes, W.A. 1998. Espécies de Triatominae (Hemiptera: Reduviidae) do Estado do Maranhão-Brasil. Cad. Saúde Públ. 14(1): 187-192.

Sherlock, J. 1979. Distribuição geográfica das principais espécies de triatomineos vetores do Trypanosoma cruzi. In: Brener, Z.; Andrade, Z., Trypanosoma cruzi e Doença de Chagas. Editora Guanabara Koogan, p. 42-88.

Silveira A.C.; Rezende D.F. 1994. Epidemiologia e controle da transmissão vetorial da doença de Chagas. Rev. Soc. Bras. Med. Trop., 27: 5-16.

Silveira A.C.; Feitosa V.R.; Borges R. 1984. Distribuição de triatomineos capturados no ambiente domiciliar, no periodo 1975/ 83, Brasil. Rev. Bras. Malariol. D. Trop. 36: $15-312$.

Zeledon, R. 1983. Vectores de la enfermedad de Chagas y sus caracteristicas ecofisiológicas. Interciencia, 8:384-396. 